\title{
A Preliminary Study for the Development of Informant-Report Scale on Cognitive-Linguistic Abilities of the Elderly: Focused on Mild Cognitive Impairment and Alzheimer's Disease
}

\author{
Mi Sook Lee', Bo Seon Kim² \\ 'Department. of Audiology and Speech-Language Pathology, Hallym University of Graduate Studies, HUGS Center for Hearing and Speech Research, \\ Seoul, Korea \\ ${ }^{2}$ Seoul Asan Rehabilitation Clinic, Incheon, Korea
}

\author{
Received: January 30, 2020 \\ Revised: February 23, 2020 \\ Accepted: February 24, 2020 \\ Correspondence: \\ Bo Seon Kim, PhD \\ Seoul Asan Rehabilitation Clinic, \\ 818 Hogupo-ro, Namdong-gu, \\ Incheon 21534, Korea \\ Tel: +82-32-466-7582 \\ Fax: +82-32-466-7581 \\ E-mail: bskimslp@gmail.com
}

\begin{abstract}
Purpose: Subjective cognitive-linguistic test is used to supplement various objective tests and increase a diagnostic accuracy. The purpose of this preliminary study was to develop and standardize the Informant-report Scale on Cognitive-Linguistic abilities of the Elderly (ISCOLE), and investigate the correlation between ISCOLE and objective test in individuals with neurological disease. Methods: People with mild cognitive impairment $(\mathrm{MCI})(n=30)$ and Alzheimer's disease (AD) $(n=32)$ completed the objective cognitive-linguistic test. Informants of these patients $(n=62)$ and controls $(n=35)$ provided ratings of ISCOLE. Results: The main findings were as follows. Firstly, there was a significant difference among three groups in all subdomains and total score of ISCOLE. Secondly, several domains like attention, higher-order cognition, and language of ISCOLE were significantly associated with the objective performances in tow groups. Thirdly, memory, language, and total score of ISCOLE were a predictor of memory in $\mathrm{MCl}$ group. AD's higher-order cognition was also predicted by attention, language and higher-order cognition of ISCOLE. Conclusion: This study is expected to help confirm the overall correlations between ISCOLE and objective test, and present the predictors of objective cognitive-linguistic performances in $\mathrm{MCl}$ and $\mathrm{AD}$. The findings have implications for the use of ISCOLE in clinical settings. We also showed evidence that the ISCOLE is an informant-based measure of cognitive-linguistic decline providing a relevant contribution to the diagnostic and prognostic investigation of patients with $\mathrm{MCl}$ and $\mathrm{AD}$.
\end{abstract}

Key Words: Alzheimer's disease, Correlation, Mild cognitive impairment, Predictor, Subjective cognitive-linguistic test.

\section{INTRODUCTION}

노화나 신경병리학적 질환은 인지적 자원의 효율적 할당을 방해하고 뇌의 기능적 용량(capacity)에 변화를 초래해 다양한 인지-의사소통의 결함을 유발한다(Braver et al., 2005; Lee \& $\mathrm{Kim}, 2019)$. 예를 들어 노인은 기억력의 저하로 다른 인지 처리 나 의사소통에 어려움을 겪기 때문에 전반적인 삶의 질이 낮아 진다(Lee, 2019). 또한 일상생활에서 다양한 주관적 호소(subjective complaints)가 발생한다. 주관적 호소란 신경학적 질환 으로 인해 인지-언어능력이 뚜렷이 저하되기 이전에 주관적으

(c) This is an Open Access article distributed under the terms of the Creative Commons Attribution Non-Commercial License (https://creativecommons.org/licenses/by-nc/4.0) which permits unrestricted non-commercial use, distribution, and reproduction in any medium, provided the original work is properly cited.
로 불편함을 느끼는 단계로서, 노인 인구의 50 60\% 정도가 경 험한다(Singh-Manoux et al., 2014). 이러한 인지-언어적 변화 는 경도인지장애(mild cognitive impairment, $\mathrm{MCI}$ ), 알츠하이 머병(Alzheimer's disease, AD) 등을 예측하는 주요 지표이므 로, 노년기부터 인지-언어능력을 지속적으로 진단할 필요가 있 다(Gavett et al., 2011; Lee, 2016a).

인지-언어적 변화를 평가하는 방식에는 몇 가지 유형이 있 다. 결함을 보이는 양상을 파악하고 중증도를 예측하기 위해 주로 객관적 및 주관적 평가를 활용한다(Lee, 2015a). 예컨대 임상가가 대상자에게 직접 실시하는 신경심리검사, 인지-언어 선별검사, 인지-언어 심화검사 등은 '객관적 평가'인 반면, 대상 자 본인이나 보호자가 관련 문항에 응답하는 형태는 주관적 평가'에 해당한다(Buelow et al., 2014; Lee, 2015b). 주관적 평 
가는 수행 주체에 따라 자기 보고형(self-report)과 정보제공자 보고형(informant-report)으로 분류된다.

객관적 평가와 달리 주관적 평가는 임상 현장에서 실시할 필 요가 없고, 일상의 기능적인 측면을 반영할 수 있다(Buelow et al., 2014). 특히 정보제공자 보고형 평가는 일상생활의 인지-언어 적 양상이나 장기간의 지속적인 변화를 관찰자의 시각에서 판단 한다(Gavett et al., 2011). 치매의 경우 중증도가 심할수록 자기 보고형 평가가 어려우므로, 정보제공자 보고형 평가의 유용성이 지속적으로 입증되고 있다(Clare et al., 2011; Lee, 2015a).

인지-언어적 변화에 대한 주관적 평가의 신뢰도를 높이려면 객관적 수행력과의 상관성을 증진하는 데 주목해야 한다. 정보 제공자 보고형이 객관적 능력을 더 잘 반영한다는 결과가 많으 나(Fyock \& Hampstead, 2015; Gavett et al., 2011; Lee, 2016b; Slavin et al., 2015), 자기 보고형의 효과성이 언급되기도 한다 (Kim et al., 2015; Martyr et al., 2014). 두 유형 모두 객관적 능 력을 잘 반영한다는 견해도 있고(Greenop et al., 2011), 인지언어의 하위 영역이나 적용 대상에 따라 다르다는 보고도 있다 (Lee, 2015a; 2016b).

Lee(2015a)의 연구에서 노인의 지남력, 기억력, 조직화 능력 은 각각 자기 보고형 평가의 문제해결력 및 언어능력과 상관성 이 크며, 정보제공자 보고형의 기억력은 객관적인 집행기능과 언어능력을 잘 반영하였다. $\mathrm{MCI}$ 의 언어능력은 자기 보고형, 전 반적 인지능력은 정보제공자 보고형이 객관적 평가와 상관성 이 높고, 치매의 기억력, 언어능력, 전반적 인지능력은 두 유형 모두 객관적 수행력을 잘 나타낸다고 보고된다(Lee, 2015a). 관 련 연구들에 대한 메타 분석(Lee, 2015a) 결과에 따르면 자기 보고형 평가는 치매 집단에서, 정보제공자 보고형은 노인, $\mathrm{MCI}$, 치매 집단 모두에서 객관적 평가와의 상관성이 높았다. 특히, 정보제공자 보고형은 $\mathrm{MCI}$ 의 변별에 유용하다는 견해도 있다(Fyock \& Hampstead, 2015).

노화와 신경학적 질환으로 인한 인지-언어적 변화를 주-객 관적 평가로 살펴보거나 양자 간의 상관성을 규명하려는 논의 가 시도되고 있지만, 주관적 평가에 대한 임상적 활용도는 매 우 제한적이다. 이의 원인은 다음과 같다. 첫째, 언어재활 현장 에서 활용할 수 있는 주관적 평가 도구가 다양하지 않을 뿐 아 니라 국내 사례를 반영한 표준화 도구가 거의 전무하기 때문이 다. 둘째, 객관적 평가에 비해 주관적 평가 도구에 대한 신뢰도 가 낮은 편이다. 이는 두 평가 유형 간의 상관성이 대부분 입증 되지 않았고, 객관적 수행력을 예측할 수 있는 증거 기반적인 자료가 부족한 데 기인한다. 셋째, 객관적 평가에 지나치게 의 존하는 경향이 있다. 특히 치매는 중증도에 따라 감정 상태의 변화나 인지-언어적 수준이 객관적 평가에 영향을 미칠 수 있 으므로(Dufouil et al., 2005; Kim et al., 2015), 정보제공자 보
고형 평가를 통해 인지-의사소통장애의 진단적 정확도를 높일 필요가 있다.

따라서 인지-의사소통장애에 대한 객관적 평가를 보완하고 진단의 정확도를 높이기 위해 정보제공자 보고형 주관적 평가 도구가 개발되어야 한다. 이는 일상생활의 수행력을 반영하므 로 신경학적 변화로 인한 인지-언어적 양상의 기능적 측면을 살펴보는 데 용이하다. 또한 인지-언어적 변화를 장기적이고 연속적으로 파악함으로써 신경병리학적 질환으로의 진전을 예 방할 수 있다. 객관적 평가나 자기 보고형 주관적 평가에 비해 임상 전 혹은 임상 단계에서 쉽게 시행할 수 있어 정확한 진단 을 내리는 데 유용하다.

본 예비연구는 노화 및 신경병리학적 질환으로 인한 인지-의 사소통장애의 주관적 평가 도구로서 노년기 인지-언어능력에 대한 정보제공자 보고형 평가척도(Informant-report Scale on Cognitive-Linguistic abilities of the Elderly, ISCOLE) (Lee, 2015b; 2016a)를 표준화하는 데 목적을 두었다. 이를 위한 구체 적인 연구 문제는 다음과 같다.

첫째, $\mathrm{MCI}$ 및 $\mathrm{AD}$, 통제 집단 간에 ISCOLE의 수행력을 비 교한다.

둘째, $\mathrm{MCI}$ 와 $\mathrm{AD}$ 집단에서 각각 $\mathrm{ISCOLE}$ 과 객관적 평가 간 의 상관성을 알아본다.

셋째, $\mathrm{ISCOLE}$ 의 하위 영역 중 $\mathrm{MCI}$ 와 $\mathrm{AD}$ 의 객관적 인지언어능력을 예측하는 변인을 제시한다.

\section{MATERIALS AND METHODS}

\section{연구 대상}

본 연구에 참여한 환자군은 $\mathrm{MCI} 30$ 명, 경도 중등도의 $\mathrm{AD}$ 32명으로 구성되었다. 이들은 수도권 소재의 대학병원 신경과, 데이케어센터, 대학 부설 언어재활센터에 내원한 환자들로부터 표집되었다. 환자군의 선정 기준으로는 1) 신경과 전문의에 의 해 $\mathrm{MCI}$ 와 $\mathrm{AD}$ 로 진단받고, 2) 임상치매척도(Clinical Dementia Rating) (Morris, 1993)에 따라 0.5, 1, 2로 분류되었으며, 3) 최 소 1년 이상의 교육을 받은 자들이었다.

인지-언어능력에 대한 주관적 평가를 위해 두 환자군의 정 보제공자가 대상군에 포함되었다. 또한 환자군과 연령 및 성별, 교육 연수의 분포를 일치시킨 정상 통제군의 정보제공자 35 명 도 참여하였다. 정보제공자는 사전 면담을 통해 대상군의 인지언어능력을 잘 파악하고 있는 자들로 선별하였다(Jorm, 2004). 사전 면담의 주요 내용으로는 1) 대상자와 동거하고 있는 가족 또는 보호자의 유무, 2) 대상자와 주 1회 이상 빈번하게 교류하 는 가족 또는 보호자의 유무, 3) 동거 또는 교류하는 자들 중 정보제공자 보고형 평가가 가능한지 여부, 4) 본인의 신경학적 
Table 1. Demographic characteristics of subjects

\begin{tabular}{lcccc}
\hline \multicolumn{1}{c}{ Characteristic } & $\mathrm{MCI}(\mathrm{n}=30)$ & $\mathrm{AD}(\mathrm{n}=32)$ & Control $(\mathrm{n}=35)$ & $\mathrm{F}$ or $\chi^{2}$ \\
\hline Age $(\mathrm{yr})$ & $75.10( \pm 3.13)$ & $74.59( \pm 5.25)$ & $72.63( \pm 4.93)$ & 2.706 \\
Gender $(\mathrm{M}: \mathrm{F})$ & $14: 16$ & $14: 18$ & $19: 16$ & 0.798 \\
Education level $(\mathrm{yr})$ & $7.93( \pm 1.98)$ & $7.91( \pm 3.43)$ & $8.00( \pm 3.61)$ & 0.008 \\
K-MMSE & $22.55( \pm 2.46)$ & $19.43( \pm 5.21)$ & $24.50( \pm 0.93)$ & $7.692^{* *}$ \\
\hline
\end{tabular}

${ }^{* *} p<0.01$. MCI: mild cognitive impairment, AD: Alzheimer's disease, K-MMSE: Korean Mini-Mental State Examination

Table 2. Demographic characteristics of informants

\begin{tabular}{lccc}
\hline \multicolumn{1}{c}{ Characteristic } & $\begin{array}{c}\text { MCI } \\
(\mathrm{n}=30)\end{array}$ & $\begin{array}{c}\mathrm{AD} \\
(\mathrm{n}=32)\end{array}$ & $\begin{array}{c}\text { Control } \\
(\mathrm{n}=35)\end{array}$ \\
\hline Age $(\mathrm{yr})$ & $55.83( \pm 13.87)$ & $54.40( \pm 13.84)$ & $68.00( \pm 13.07)$ \\
Gender $(\mathrm{M}: \mathrm{F})$ & $16: 14$ & $11: 21$ & $16: 19$ \\
Education level $(\mathrm{yr})$ & $10.77( \pm 3.13)$ & $11.06( \pm 2.70)$ & $8.80( \pm 2.88)$ \\
Type & & & \\
$\quad$ Spouse & 9 & 10 & 29 \\
Child & 11 & 19 & 6 \\
Caregiver & 9 & 2 & - \\
Others & 1 & 1 & - \\
\hline
\end{tabular}

MCI: mild cognitive impairment, AD: Alzheimer's disease

병력 및 정상적인 인지능력에 대한 확인, 5) 대상군에 대한 인구 통계학적 정보의 수집 등이었다. 이를 통해 선별된 정보제공자에 는 대상자의 가족(배우자, 자녀 등), 간병인, 친척이 포함되었다.

정상 노인, $\mathrm{MCI}, \mathrm{AD}$ 집단 간에 연령, 성별, 교육 연수, 한국형 간이정신상태검사(Korean Mini-Mental State Examination, K$\mathrm{MMSE})$ 의 분포에 대한 동질성 검정 결과 $\mathrm{K}-\mathrm{MMSE}(\mathrm{F}=7.692$, $p<0.01)$ 를 제외하고 연령 $(\mathrm{F}=2.706, p=0.072)$, 성별 $\left(\chi^{2}=\right.$ $0.798, p=0.671)$, 교육 연수 $(\mathrm{F}=0.008, p=0.992)$ 의 분포상 유 의미한 차이는 없었다.

세 집단의 대상군 및 정보제공자의 연령, 성별, 교육 연수, $\mathrm{K}-$ MMSE 등 인구통계학적 특성은 Table 1과 2에 각각 제시하였다.

\section{연구 도구}

세 집단의 인지-언어능력에 대해 정보제공자가 주관적으로 평정한 결과를 알아보기 위해 ISCOLE을 시행하였다. ISCOLE은 주의력(지속 및 분리 주의력), 지남력(사람/시간/장소), 시지각력(시공간력/시각 구성력), 기억력(장기/단기/즉각 기억 및 학습능력), 조직화 능력(범주화/순서화), 추론력(귀납적 및 수렴적 사고), 문제해결력(문제 추론/의사 결정), 집행기능(계획 화/실행화), 언어능력(이해/표현/이름 대기/읽기/쓰기/계산/화 용) 등 9 개 하위 영역의 27 개 문항으로 구성되어 있다. 대상자 의 인지-언어능력을 1 년 전과 비교하여 '아니다(0)-약간 그렇 다(1)-보통이다(2)-많이 그렇다(3)-항상 그렇다(4)'의 5점 척도 로 평정한다. 점수가 높을수록 인지-언어능력이 낮은 것으로 해석된다.
환자군의 인지-언어능력에 대한 객관적 평가 도구로서 인 지-의사소통장애 간편검사(Brief test of Cognitive-Communication Disorders, BCCD) (Lee \& Kim, 2019)를 사용하였다. $\mathrm{BCCD}$ 는 인지 15 개, 의사소통 12 개 등 총 27 개 문항으로 구성 되어 있다. 인지의 하위 검사에는 주의력(선택 주의력/분리 주 의력), 시공간력(시공간 구성), 기억력(구어 및 비구어 작업기억/ 회상과 재인), 고차원 인지(조직화 능력/추론력/문제해결력/집 행기능) 등 4 개 영역이 포함된다. 의사소통의 4 개 하위 영역은 이해(비유 언어/상징 및 기호), 표현(단어 유창성/단어 정의/비 유 언어), 읽기(읽기 이해) 및 쓰기(받아쓰기), 화용언어(담화/화 용 표현) 등으로 구성된다. 화용언어의 담화 문항을 제외한 나 머지는 모두 문항당 0 2점으로 채점하며, 담화 문항은 채점 기 준에 따라 최대 8점을 부여한다.

ISCOLE과 $\mathrm{BCCD}$ 의 주요 구성은 Table 3에 제시하였다.

\section{연구 절차}

세 집단의 정보제공자에게 ISCOLE을 적용하여 인지-언어 능력에 대한 주관적 평가를 실시하였다. 정보제공자로서의 적 합성을 판별하기 위해 사전 면담을 시행한 후 ISCOLE의 27개 문항을 평정하도록 하였다. 평정 방법을 숙지한 후 정보제공자 가 직접 시행한 경우가 대부분이었으나, 신체적 문제(시력, 팔의 움직임 등), 개별적 요청 등이 있을 시 연구자가 문항을 읽어 주 고 답변하도록 하였다. ISCOLE의 평균 소요시간은 약 5 10분 이었다.

환자군의 객관적인 인지-언어능력을 알아보기 위해 $\mathrm{BCCD}$ 를 적용하였다. 이에 앞서 환자 또는 정보제공자를 대상으로 인구통 계학적 및 신경학적 정보, 기타 능력(청력, 시력)에 대한 사례 면 담을 실시하였다. 본 검사는 소음이 최소화된 조용한 방에서 일 대일 직접 평가 방식으로 진행되었다. 문항의 특성에 따라 검사자 가 기록지에 직접 기록하거나 피검자가 반응지에 표기하였고, 담 화 문항을 분석하기 위해 자발화를 녹음하였다. 검사 소요시간 은 평균 20 30분이었다. 담화를 제외한 모든 문항은 명시된 채 점 기준에 따라 3 점 척도 $(2,1,0)$ 로 점수를 산정하였다. 담화 문 항은 규정된 분석 틀에 따라 응집성 및 통일성은 각각 0 3점, 명 제 및 심은 0 2점으로 평정하여 최대 8점을 부여하였다. $\mathrm{BCCD}$ 의 점수가 높을수록 인지-언어능력이 양호함을 의미한다. 
Table 3. Construction of ISCOLE and BCCD

\begin{tabular}{lll}
\hline \multicolumn{1}{c}{ Domain } & \multicolumn{1}{c}{ ISCOLE $\left(27^{*}\right)$} & BCCD $\left(27^{*}\right)$ \\
\hline Cognition & & Selective/divided (2) \\
Attention & Sustained/divided (2) & - \\
Orientation & Person/time/place (3) & Visuospatial (1) \\
Visuoperception & Visuospatial/visuoconstruction (2) & WM/recall etc. (3) \\
Memory & LTM/learning etc. (4) & Categorization etc. (2) \\
Organization & Categorization etc. (2) & Divergent etc. (2) \\
Reasoning & Convergent/inductive (2) & Inferences etc. (3) \\
Problem solving & Inferences etc. (2) & Updating etc. (2) \\
Executive function & Activating/planning (2) & Pragmatic etc. (12) \\
Communication & & Expression/naming etc. (8) \\
Language & &
\end{tabular}

*a number of items. ISCOLE: Informant-report Scale on Cognitive-Linguistic abilities of the Elderly, BCCD: Brief test of Cognitive-Communication Disorders, LTM: long-term memory, WM: working memory

Table 4. Performances of Informant-report Scale on Cognitive-Linguistic abilities of the Elderly in three group

\begin{tabular}{lcccc}
\hline \multicolumn{1}{c}{ Characteristic } & Control & MCI & AD & F \\
\hline Attention & $0.97( \pm 0.92)$ & $3.07( \pm 1.53)$ & $3.06( \pm 2.00)$ & $20.871^{* *}$ \\
Orientation & $0.23( \pm 0.43)$ & $2.43( \pm 1.01)$ & $3.00( \pm 2.46)$ & $30.378^{* *}$ \\
Visuoperception & $0.00( \pm 0.00)$ & $1.53( \pm 1.20)$ & $1.94( \pm 1.72)$ & $24.818^{* *}$ \\
Memory & $1.74( \pm 0.92)$ & $5.77( \pm 2.67)$ & $6.97( \pm 3.67)$ & $36.339^{* *}$ \\
Organization & $0.00( \pm 0.00)$ & $2.50( \pm 1.48)$ & $3.19( \pm 1.77)$ & $55.578^{* *}$ \\
Reasoning & $0.11( \pm 0.32)$ & $2.87( \pm 1.68)$ & $3.88( \pm 1.68)$ & $69.574^{* *}$ \\
Problem solving & $0.34( \pm 0.64)$ & $1.97( \pm 0.89)$ & $3.91( \pm 1.89)$ & $67.643^{* *}$ \\
Executive function & $0.29( \pm 0.57)$ & $1.37( \pm 1.10)$ & $3.03( \pm 1.58)$ & $48.539^{* *}$ \\
Language & $1.06( \pm 1.39)$ & $9.97( \pm 4.34)$ & $12.13( \pm 5.49)$ & $70.507^{* *}$ \\
Total score & $4.74( \pm 2.64)$ & $31.47( \pm 9.28)$ & $41.09( \pm 16.83)$ & $97.539^{* *}$ \\
\hline
\end{tabular}

${ }^{* *} p<0.01$. MCI: mild cognitive impairment, AD: Alzheimer's disease

통계 분석

본 연구에서는 통계 분석 프로그램으로 SPSS 21.0 version (IBM Corp., Armonk, NY, USA)을 활용하였다. 연령과 성별, 교육 연수, $\mathrm{K}-\mathrm{MMSE}$ 점수상 집단 간 분포에 차이가 있는지를 확인하기 위해 일원배치 분산분석(one-way analysis of variance)과 카이제곱 검정 $(\chi 2$ test)을 사용하였다. 기술통계 및 일 원배치 분산분석을 통해 세 집단별로 ISCOLE의 수행력을 비 교하였다. 환자군에서 ISCOLE과 객관적 수행력 간의 상관성 은 피어슨 상관계수(Pearson correlation coefficient) 분석으로 알아보았다. 객관적 인지-언어능력에 대한 예측변수는 단계적 다중선형 회귀분석(stepwise multiple linear regression analysis)에 근거해 제시하였다.

\section{RESULTS}

\section{ISCOLE의 수행력 비교}

두 환자군과 통제군에서 ISCOLE의 수행력에 차이가 있는 지를 비교한 결과는 Table 4에 제시하였다.
ISCOLE의 모든 하위 영역 및 총점에서 정상 통제군에 비해 두 환자군의 수행력이 낮게 나타났다. 세 집단 간의 차이는 모 든 하위 영역과 총점에서 유의미하였다 $(p<0.01)$. 사후검정 결 과 추론력, 문제해결력, 집행기능 등 3 개 영역과 총점은 통제군 과 $\mathrm{MCI}$, 통제군과 $\mathrm{AD}, \mathrm{MCl}$ 와 $\mathrm{AD}$ 등 모든 두 집단 간에 유의 미한 차이가 있었다 $(p<0.01$ 또는 $p<0.05)$. 주의력, 언어 등 나머지 하위 영역은 통제군과 $\mathrm{MCI}$, 통제군과 $\mathrm{AD}$ 집단 간의 차 이가 유의미하였다 $(p<0.01)$.

\section{$\mathrm{MCI}$ 및 $\mathrm{AD}$ 의 ISCOLE과 객관적 수행력 간 상관성}

두 환자군의 주-객관적 평가에서 각 하위 영역 및 총점 간의 상관성을 알아보았다(Table 5, 6). 두 평가의 하위 영역을 상호 분석하기 위해 ISCOLE의 조직화 능력, 추론력, 문제해결력, 집 행기능은 각 점수를 합산하여 $\mathrm{BCCD}$ 의 고차원 인지 영역과 비 교하였다. $\mathrm{BCCD}$ 에 포함되지 않은 지남력은 분석에서 제외하였 고, $\mathrm{ISCOLE}$ 의 언어와 $\mathrm{BCCD}$ 의 의사소통 영역을 대응하여 비 교하였다.

$\mathrm{MCI}$ 집단에서 주관적 평가의 주의력, 고차원 인지, 언어, 총 
Table 5. Correlation between subjective and objective tests in mild cognitive impairment group

\begin{tabular}{lcccccc}
\hline & \multicolumn{5}{c}{ Subjective test (ISCOLE) } \\
\cline { 2 - 7 } & Attention & VP & Memory & HC & Language & TS \\
\hline Objective test (BCCD) & & & & & \\
Attention & $-0.941^{* *}$ & 0.142 & -0.029 & $-0.570^{* *}$ & $-0.745^{* *}$ & $-0.765^{* *}$ \\
VP & 0.163 & $-0.605^{* *}$ & -0.199 & 0.032 & 0.180 & -0.044 \\
Memory & -0.074 & -0.356 & $-0.696^{* *}$ & -0.014 & -0.017 & -0.291 \\
HC & $-0.842^{* *}$ & 0.312 & 0.014 & $-0.779^{* *}$ & $-0.822^{* *}$ & $-0.836^{* *}$ \\
Communication & $-0.780^{* *}$ & 0.268 & -0.115 & $-0.514^{* *}$ & $-0.868^{* *}$ & $-0.790^{* *}$ \\
TS & $-0.874^{* *}$ & 0.177 & -0.161 & $-0.686^{* *}$ & $-0.840^{* *}$ & $-0.888^{* *}$ \\
\hline
\end{tabular}

${ }^{* *} p<0.01$. ISCOLE: Informant-report Scale on Cognitive-Linguistic abilities of the Elderly, BCCD: Brief test of Cognitive-Communication Disorders, VP: visuoperception, HC: higher-order cognition, TS: total score

Table 6. Correlation between subjective and objective tests in Alzheimer's disease group

\begin{tabular}{lllllll}
\hline & \multicolumn{5}{c}{ Subjective test (ISCOLE) } \\
\cline { 2 - 6 } & Attention & VP & Memory & HC & Language & TS \\
\hline Objective test (BCCD) & & & & & \\
Attention & $-0.597^{* *}$ & $-0.522^{* *}$ & -0.076 & $-0.361^{*}$ & $-0.469^{* *}$ & $-0.490^{* *}$ \\
VP & -0.335 & $-0.648^{* *}$ & 0.032 & -0.150 & -0.340 & -0.290 \\
Memory & -0.201 & -0.215 & $-0.577^{* *}$ & -0.289 & $-0.490^{* *}$ & $-0.411^{*}$ \\
HC & $-0.396^{*}$ & $-0.443^{*}$ & -0.261 & $-0.666^{* *}$ & $-0.681^{* *}$ & $-0.662^{* *}$ \\
Communication & $-0.354^{*}$ & $-0.460^{* *}$ & -0.318 & $-0.532^{* *}$ & $-0.699^{* *}$ & $-0.624^{* *}$ \\
TS & $-0.426^{*}$ & $-0.503^{* *}$ & -0.341 & $-0.561^{* *}$ & $-0.707^{* *}$ & $-0.651^{* *}$ \\
\hline
\end{tabular}

${ }^{*} p<0.05,{ }^{* *} p<0.01$. ISCOLE: Informant-report Scale on Cognitive-Linguistic abilities of the Elderly, BCCD: Brief test of Cognitive-Communication Disorders, VP: visuoperception, HC: higher-order cognition, TS: total score

Table 7. Predictors of objective cognitive-linguistic abilities in mild cognitive impairment group

\begin{tabular}{lcc}
\hline \multirow{2}{*}{ Objective } & \multicolumn{2}{c}{ Subjective test (ISCOLE) } \\
\cline { 2 - 3 } cognitive-linguistic abilities & \multicolumn{1}{c}{ Predictors } & $\beta$-value \\
\hline Attention & - & - \\
Visuoperception & Visuoperception & $-0.938^{* *}$ \\
Memory & Memory & $-1.109^{* *}$ \\
& Language & $-1.769^{* *}$ \\
& Total score & $3.487^{*}$ \\
Higher-order cognition & - & - \\
Communication & - & - \\
Total score & - & - \\
\hline
\end{tabular}

${ }^{*} p<0.05,{ }^{* *} p<0.01$. ISCOLE: Informant-report Scale on Cognitive-Linguistic abilities of the Elderly

점은 공통적으로 객관적 평가의 주의력, 고차원 인지, 의사소 통, 총점과 유의미한 부적 상관성을 보였다 $(p<0.01)$. 시지각력 과 기억력은 두 평가의 동일 영역끼리 각각 유의미한 상관성이 있었다 $(p<0.01)$.

$\mathrm{AD}$ 집단의 경우 주관적 평가의 언어와 총점은 모두 객관적 평 가의 주의력, 기억력, 고차원 인지, 의사소통, 총점 등 5개 영역과 상관관계가 유의미하였다 $(p<0.01$ 또는 $p<0.05)$. 또한 주관적 평가의 주의력과 고차원 인지는 공통적으로 객관적 평가의 주의 력, 고차원 인지, 의사소통, 총점과 유의미한 부적 상관을 보였고
Table 8. Predictors of objective cognitive-linguistic abilities in Alzheimer's disease group

\begin{tabular}{llc}
\hline \multirow{2}{*}{$\begin{array}{c}\text { Objective } \\
\text { cognitive-linguistic abilities }\end{array}$} & \multicolumn{2}{c}{ Subjective test (ISCOLE) } \\
\cline { 2 - 3 } Attention & Attention & $\beta$-value \\
& Higher-order cognition & $-1.177^{* *}$ \\
Visuoperception & \multicolumn{1}{c}{$-0.726^{*}$} \\
Memory & \multicolumn{1}{c}{-} & - \\
Higher-order cognition & Attention & - \\
& Language & $-0.701^{* *}$ \\
& Higher-order cognition & $-1.167^{* *}$ \\
& Total score & $1.791^{*}$ \\
Communication & & - \\
Total score & Attention & $-0.672^{* *}$ \\
& Higher-order cognition & $-0.864^{*}$ \\
\hline
\end{tabular}

${ }^{*} p<0.05,{ }^{* *} p<0.01$. ISCOLE: Informant-report Scale on Cognitive-Linguistic abilities of the Elderly

$(p<0.01$ 또는 $p<0.05)$, 시지각력은 기억력을 제외한 나머지 5 개 영역과 모두 유의한 상관성이 있었다 $(p<0.01$ 또는 $p<0.05)$. 두 평가의 기억력 간에도 상관성이 유의미하였다 $(p<0.01)$.

\section{객관적 인지-언어능력의 예측변인}

주관적 평가인 ISCOLE에서 두 환자군의 객관적 인지-언어 
능력을 잘 반영하는 하위 영역이 무엇인지를 분석한 결과는 Table 7과 8에 각각 제시하였다.

$\mathrm{MCI}$ 의 시지각력은 ISCOLE의 시지각력이 주요 예측변인이 며 $(p<0.01)$, 기억력은 ISCOLE의 기억력 $(p<0.01)$, 언어 $(p<$ $0.01)$, 총점 $(p<0.05)$ 을 통해 가장 잘 반영되는 것으로 나타났다. $\mathrm{AD}$ 의 주의력 및 총점은 공통적으로 ISCOLE의 주의력 $(p<$ $0.01)$ 과 고차원 인지 $(p<0.05)$ 를 통해 민감하게 반영되며, 고차 원 인지는 주의력 $(p<0.01)$, 언어 $(p<0.05)$, 고차원 인지 $(p<$ $0.01)$, 총점 $(p<0.05)$ 이 주요 예측변인으로 분석되었다.

\section{DISCUSSIONS}

본 연구는 인지-의사소통장애의 객관적 평가를 보완하고 진 단적 정확도를 높이는 데 활용할 정보제공자 보고형 평가 도구 로서 ISCOLE을 개발하고 표준화하기 위한 예비연구이다. 먼저 $\mathrm{MCI}$ 및 $\mathrm{AD}$ 가 포함된 환자군과 정상 통제군 간에 ISCOLE의 수행력을 비교하였다. 또한 두 환자군에서 ISCOLE과 객관적 평가 간에 어떠한 상관성이 있는지를 알아보고, $\mathrm{MCI}$ 와 $\mathrm{AD}$ 의 객관적 인지-언어능력을 예측하는 ISCOLE의 하위 영역을 제 시하였다.

본 연구에서 가족, 간병인 등의 정보제공자를 통해 평가한 ISCOLE의 수행력은 정상 통제군에 비해 두 환자군의 수행력 이 낮게 나타났다. 즉, 정상군에서 $\mathrm{MCI}, \mathrm{AD}$ 집단으로 갈수록 대부분의 하위 영역과 총점에서 수행력이 저하되었으며, 특히 추론력, 문제해결력, 집행기능 등 3 개 영역과 총점은 모든 두 집 단 간에 유의미한 차이가 있었다.

정상 노인의 절반 이상이 인지-언어에 대한 주관적 호소를 경험하나, MCI의 주관적 호소율은 20.6\%에 불과하다(Jungwirth et al., 2005; Singh-Manoux et al., 2014). 이 같은 비율은 인지-언어 및 기능적 상태에 대한 인식이 저하되는 $\mathrm{AD}$ 의 경우 더욱 급감한다(Buelow et al., 2014). 따라서 $\mathrm{MCl}$ 와 $\mathrm{AD}$ 의 정보 제공자는 이들의 인지-언어능력에 대한 주요 평가자로서 신뢰 할 만하다. 실제로 정상 노인과 $\mathrm{MCI}, \mathrm{AD}$ 의 정보제공자형 평가 에서 유의미한 수행력의 차이가 다수 보고된 바 있다(Farias et al., 2005; Isella et al., 2006; Martyr et al., 2014).

추론력, 문제해결력, 집행기능과 같은 고차원 인지는 다영역 적 처리를 기반으로 하는 복합적인 능력으로 주의력, 기억력, 인 지 처리 속도, 작업기억 등과의 연계성 때문에 신경학적 변화에 특히 민감하다(Emery et al., 2008; Lee, 2016c; Lee \& Kim, 2019; Salthouse, 2000). 추론력, 문제해결력, 집행기능이 정상군 과 $\mathrm{MCI}$, 정상군과 $\mathrm{AD}, \mathrm{MCI}$ 와 $\mathrm{AD}$ 간을 변별하는 데 모두 유 의하다는 본 연구 결과는 고차원적 인지의 이 같은 속성을 직 접적으로 반영한다.
두 환자군에서 주-객관적 수행력 간의 상관성을 분석한 결 과는 하위 영역 및 집단에 따라 다소 차이를 보였다. $\mathrm{MCI}$ 는 주 관적 평가의 주의력, 고차원 인지, 언어, 총점이 객관적 평가의 주의력, 고차원 인지 등 4개 영역과 유의한 상관이 있었다. $\mathrm{AD}$ 는 주관적 평가의 언어와 총점이 객관적 평가의 기억력, 의사소 통 등 5개 영역과, 그리고 주관적 평가의 주의력, 시지각력, 고 차원 인지는 객관적 평가의 주의력, 의사소통 등 4 개 영역과 유 의미한 상관성을 보였다. 특히, 두 집단의 주-객관적 평가에서 주의력, 고차원 인지, 언어/의사소통 및 총점이 공통적으로 다 수 영역에 걸쳐 상관관계가 유의미하였다.

$\mathrm{MCI}$ 와 $\mathrm{AD}$ 를 대상으로 주관적 및 객관적 인지-언어능력 간 의 상관성을 하위 영역별로 살펴본 연구는 거의 드물다. 즉, 특 정 평가 척도의 총점을 비교하거나 자기 보고형, 정보제공자 보 고형 등 평가 주체에 따른 차이에 국한한 연구들이 대부분이다 (Farias et al., 2005; Gavett et al., 2011; Isella et al., 2006). 주 관적 호소의 주 영역인 기억력에 중점을 둔 선행연구도 있다. 예 컨대 Buelow et al.(2014)은 단어 학습, 지연 회상 등 기억의 하 위 유형에 대한 정보제공자 보고형 평가가 $\mathrm{MCI}$ 와 $\mathrm{AD}$ 의 객관 적 수행력과 상관성이 있음을 규명했다. 정상 노인을 대상으로 한 연구에서는 주관적 평가의 기억력, 언어, 인지-언어 총점이 객관적 평가의 여러 하위 영역과 상관성을 보였다(Lee, 2016b). 특히 정보제공자가 평가한 언어능력이 객관적 평가의 기억력, 고 차원 인지(조직화 능력, 추론력, 문제해결력), 언어, 총점 등 다수 영역과 높은 상관성을 보인 것은 본 연구 결과와도 일치한다.

본 연구에서는 $\mathrm{MCI}$ 에 비해 $\mathrm{AD}$ 의 주-객관적 평가에 있어 보다 광범위한 하위 영역 간의 상관성이 입증되었다. 환자의 유 형별로 나타나는 이 같은 양상은 연구마다 다소 차이를 보인다. 예를 들어 기억상실형(amnestic) $\mathrm{MCI}$ 와 비교할 때 비기억상실 형(nonamnestic) $\mathrm{MCI}$ 의 기억력은 양자 간에 유의미한 상관성 이 없다(Farias et al., 2005). 이 밖에 주-객관적 평가의 상관성 을 통해 $\mathrm{MCI}$ 에서 $\mathrm{AD}$ 로의 전환 여부를 변별할 수 있다는 주장 도 있다(Estévez-González et al., 2003; Isella et al., 2006). 또 한 초기 $\mathrm{AD}$, 잠정적(probable) $\mathrm{AD}$ 등 $\mathrm{AD}$ 의 세부 유형에 초점 을 맞추고 다양한 유형의 주-객관적 평가를 시행함으로써 정보 제공자 보고형 척도의 신뢰성을 검증하기도 한다(Martyr et al., 2014; Tierney et al., 2003). 이들이 주로 주의력, 기억력, 언어 등 을 포함한 전반적인 인지-언어 수행력에 근거한 점을 고려할 때 $\mathrm{AD}$ 집단에서 다수의 하위 영역 및 총점 간에 상관성을 보인 본 연구 결과를 뒷받침한다. 특히 다른 인지-언어 수행의 기초 로 작용하는 주의력, 복잡하고 다영역적인 처리에 기반한 고차 원 인지 및 언어 능력, 전반적 수행력에 해당하는 인지-언어 총 점 등의 영역별 속성도 반영된 것으로 추측된다.

마지막으로 $\mathrm{MCI}$ 와 $\mathrm{AD}$ 의 객관적 인지-언어능력에 대한 예 
측변인을 분석한 결과 $\mathrm{MCI}$ 의 기억력은 ISCOLE의 기억력, 언 어, 총점을 통해 가장 잘 예측되었다. 이와 유사하게 주관적 평가의 즉각 및 지연 기억력이 $\mathrm{MCI}$ 의 예측변인으로 간주되기 도 한다(Isella et al., 2006). 실제로 기억력에 대해 주-객관적 평가 간의 상호 예측성을 살펴본 연구들이 비교적 많은 편이다 (Fyock \& Hampstead, 2015; Gifford et al., 2015; Martyr et al., 2014; Schmitter-Edgecombe et al., 2012). Lee(2015a)의 메타 분석 연구에서는 $\mathrm{MCI}$ 에 비해 정상 노인이나 치매 집단의 기억력이 주관적 평가를 통해 잘 예측된다고 보고된 바 있으나, 대다수 연구에서 $\mathrm{MCI}$ 의 기억력은 주관적 인지-언어능력의 주 요 예측변인으로 꼽힌다(Edmonds et al., 2014; Farias et al., 2005; Li et al., 2012).

본 연구에서 $\mathrm{AD}$ 의 객관적 수행력 중 주의력 및 총점은 ISCOLE의 주의력과 고차원 인지, 그리고 고차원 인지는 ISCOLE의 주의력, 언어, 고차원 인지가 주요 예측변인이었다. $\mathrm{MCI}$ 와 $\mathrm{AD}$ 를 변별하는 주요 예측인자로서 흔히 주관적 평가 의 주의력, 지남력, 기억력, 언어, 전반적 인지-언어 등이 제안된 다(Buelow et al., 2014; Farias et al., 2005). 관련 선행연구를 분석한 결과에서도 $\mathrm{AD}$ 의 언어와 전반적 인지능력이 주관적 평가 결과를 잘 드러낸다고 보고하였다(Lee, 2015a). 이들은 특 히 자기 보고형에 비해 정보제공자 보고형 평가에서 신뢰도가 더 높다(Bruce et al., 2008; Lenehan et al., 2012). 이는 본 연 구에서 ISCOLE의 주의력, 언어, 고차원 인지 등이 $\mathrm{AD}$ 의 객관 적 수행력을 민감하게 반영한 결과와 일맥상통한다.

요컨대 본 연구는 인지-의사소통장애의 주관적 평가 도구로 서 개발한 $\mathrm{ISCOLE}$ 을 $\mathrm{MCI}$ 와 $\mathrm{AD}$ 환자군에 직접 적용함으로 써 객관적 수행력과의 상관성을 확인하고 유용한 예측변인을 제시하고자 하였다. 이를 통해 객관적 인지-언어 평가를 보완 하고 진단적 정확도를 높이는 데 활용 가능한 정보제공자 보고 형 평가의 효용성을 입증하였다.

본 연구의 제한점과 향후 연구를 위한 제언은 다음과 같다. 첫째, 주-객관적 평가 간의 상관성을 입증하기 위해 보다 다양 한 인지-언어 검사와 비교할 필요가 있다. 본 연구에서는 현재 개발 중인 객관적 평가 도구를 활용하였으나, 추후에는 신뢰도 와 타당도가 입증된 다른 검사를 추가함으로써 주관적 평가 도구로서의 신뢰도를 높이는 데 기여하고자 한다. 둘째, 본 연 구에서는 두 환자군과 통제군의 정보제공자 유형이 균일하지 않았다. 예를 들어 $\mathrm{MCI}$ 와 $\mathrm{AD}$ 집단은 배우자와 자녀, 간병인의 비중이 균일하나 통제군은 배우자의 비중이 대다수를 차지한 다. 정보제공자에 대한 객관화된 기준이 없는 점을 고려해, 본 연구에서는 대상군과 주 1 회 이상 빈번하게 교류하는 자 등으 로 정보제공자를 제한하였다. 그럼에도 불구하고 정보제공자의 유형별로 평정에 차이가 발생할 수 있으므로 이에 대한 보완이
필요할 것이다. 셋째, 향후에는 다수의 정상군과 환자군을 추가 하여 ISCOLE의 정상 규준을 제시하고 신뢰도와 타당도를 다 양하게 검증할 것이다.

중심 단어 : 알츠하이머병·상관성·경도인지장애· 예측변인·주관적 인지-언어 평가.

\section{Ethical Statement}

We explained exactly the purpose and process of this study to all participants in advance. And they agreed to participate in it. This study was approved by the Institutional Review Board (\#HUGSAUD461850, \#201908-010-002).

\section{Acknowledgments}

The authors thank to the participants.

\section{Declaration of Conflicting Interests}

There are no conflict of interests.

\section{Funding}

N/A.

\section{Author Contributions}

All authors contributed equally to this work. M.L. designed and performed experiments, analyzed data, and wrote the paper; M.L. and B.K. designed and performed experiments in the clinic; M.L. provided statistical analysis and critical revision. Also, the authors discussed the results together and implications and commented on the manuscript at each stage.

\section{ORCID iDs}

Mi Sook Lee

Bo Seon Kim

https://orcid.org/0000-0001-9255-565X
https://orcid.org/0000-0002-8351-1417

\section{REFERENCES}

Braver, T. S., Satpute, A. B., Rush, B. K., Racine, C. A., \& Barch, D. M. (2005). Context processing and context maintenance in healthy aging and early stage dementia of the Alzheimer's type. Psychology and Aging, 20(1), 33-46.

Bruce, J. M., Bhalla, R., Westervelt, H. J., Davis, J., Williams, V., \& Tremont, G. (2008). Neuropsychological correlates of self-reported depression and self-reported cognition among patients with mild cognitive impairment. Journal of Geriatric Psychiatry and Neurology, 21(1), 34-40.

Buelow, M. T., Tremont, G., Frakey, L. L., Grace, J., \& Ott, B. R. (2014). Utility of the cognitive difficulties scale and association with objective test performance. American Journal of Alzheimer's Disease and Other Dementias, 29(8), 755-761.

Clare, L., Marková, I. S., Roth, I., \& Morris, R. G. (2011). Awareness in Alzheimer's disease and associated dementias: Theoretical framework and clinical implications. Aging and Mental Health, 15(8), 936-944.

Dufouil, C., Fuhrer, R., \& Alpérovitch, A. (2005). Subjective cognitive complaints and cognitive decline: Consequence or predictor? The epidemiology of vascular aging study. Journal of the American Geriatrics Society, 53(4), 616-621.

Edmonds, E. C., Delano-Wood, L., Galasko, D. R., Salmon, D. P., \& Bondi, M. W. (2014). Subjective cognitive complaints contribute to misdiagnosis of mild cognitive impairment. Journal of the International Neuropsychological Society, 20(8), 836-847.

Emery, L., Hale, S., \& Myerson, J. (2008). Age differences in proactive interference, working memory, and abstract reasoning. Psychology and Ag- 
ing, 23(3), 634-645.

Estévez-González, A., Kulisevsky, J., Boltes, A., Otermín, P., \& García-Sánchez, C. (2003). Rey verbal learning test is a useful tool for differential diagnosis in the preclinical phase of Alzheimer's disease: Comparison with mild cognitive impairment and normal aging. International Journal of Geriatric Psychiatry, 18(11), 1021-1028.

Farias, S. T., Mungas, D., \& Jagust, W. (2005). Degree of discrepancy between self and other-reported everyday functioning by cognitive status: Dementia, mild cognitive impairment, and healthy elders. International Journal of Geriatric Psychiatry, 20(9), 827-834.

Fyock, C. A. \& Hampstead, B. M. (2015). Comparing the relationship between subjective memory complaints, objective memory performance, and medial temporal lobe volumes in patients with mild cognitive impairment. Alzheimer's and Dementia: Diagnosis, Assessment and Disease Monitoring, 1(2), 242-248.

Gavett, R. A., Dunn, J. E., Stoddard, A., Harty, B., \& Weintraub, S. (2011). The Cognitive Change in Women Study (CCW): Informant ratings of cognitive change but not self ratings are associated with neuropsychological performance over three years. Alzheimer Disease and Associated Disorders, 25(4), 305-311.

Gifford, K. A., Liu, D., Damon, S. M., Chapman, W. G. IV., Romano, R. R. III., Samuels, L. R., et al.; Alzheimer's Disease Neuroimaging Initiative. (2015). Subjective memory complaint only relates to verbal episodic memory performance in mild cognitive impairment. Journal of $\mathrm{Al}$ zheimer's Disease, 44(1), 309-318.

Greenop, K. R., Xiao, J., Almeida, O. P., Flicker, L., Beer, C., Foster, J. K., et al. (2011). Awareness of cognitive deficits in older adults with cognitive-impairment-no-dementia (CIND): Comparison with informant report. Alzheimer Disease and Associated Disorders, 25(1), 24-33.

Isella, V., Villa, L., Russo, A., Regazzoni, R., Ferrarese, C., \& Appollonio, I. M. (2006). Discriminative and predictive power of an informant report in mild cognitive impairment. Journal of Neurology, Neurosurgery, and Psychiatry, 77(2), 166-171.

Jorm, A. F. (2004). The informant questionnaire on cognitive decline in the elderly (IQCODE): A review. International Psychogeriatrics, 16(3), 275293.

Jungwirth, S., Weissgram, S., Zehetmayer, S., Tragl, K. H., \& Fischer, P. (2005). VITA: Subtypes of mild cognitive impairment in a community-based cohort at the age of 75 years. International Journal of Geriatric Psychiatry, 20(5), 452-458.

Kim, B. S., Lee, M. S., \& Kim, H. (2015). Subjective language complaints: Are they reflected in objective language test performance? Communication Sciences and Disorders, 20(2), 214-221.

Lee, M. S. (2015a). Meta-analysis of correlation between subjective and objective cognitive-linguistic tests: Focused on normal aging, MCI, and dementia. Journal of the Korea Academia-Industrial Cooperation Society, 16(11), 7414-7423.

Lee, M. S. (2015b). A study of the content validity of informant-reported scale of cognitive-linguistic abilities of the elderly (ISCOLE). Journal of Speech and Hearing Disorders, 24(4), 275-286.

Lee, M. S. (2016a). Reliability and validity of informant-report scale on cognitive-linguistic abilities of the elderly (ISCOLE). Communication Sciences and Disorders, 21(1), 151-161.

Lee, M. S. (2016b). Correlation between subjective and objective cognitivelinguistic tests in older adults. Journal of the Korea Academia-Industrial cooperation Society, 17(5), 548-556.

Lee, M. S. (2016c). Correlation between pragmatic language and executive function in patients with acquired brain injury. The Journal of the Korea Contents Association, 16(5), 58-67.

Lee, M. S. (2019). Factors affecting quality of communication life in older adults: Focused on the relationship with quality of life, cognition, and psychoemotional aspects. Audiology and Speech Research, 15(3), 232240.

Lee, M. S. \& Kim, B. S. (2019). A preliminary study for the development of brief test of cognitive-communication disorders (BCCD). Journal of Special Education and Rehabilitation Science, 58(4), 321-344.

Lenehan, M. E., Klekociuk, S. Z., \& Summers, M. J. (2012). Absence of a relationship between subjective memory complaint and objective memory impairment in mild cognitive impairment (MCI): Is it time to abandon subjective memory complaint as an MCI diagnostic criterion? International Psychogeriatrics, 24(9), 1505-1514.

Li, F., Jia, X. F., \& Jia, J. (2012). The informant questionnaire on cognitive decline in the elderly individuals in screening mild cognitive impairment with or without functional impairment. Journal of Geriatric Psychiatry and Neurology, 25(4), 227-232.

Martyr, A., Nelis, S. M., \& Clare, L. (2014). Predictors of perceived functional ability in early-stage dementia: Self-ratings, informant ratings and discrepancy scores. International Journal of Geriatric Psychiatry, 29(8), 852-862.

Morris, J. C. (1993). The clinical dementia rating (CDR): Current version and scoring rules. Neurology, 43(11), 2412-2414.

Salthouse, T. A. (2000). Item analyses of age relations on reasoning tests. Psychology and Aging, 15(1), 3-8.

Schmitter-Edgecombe, M., McAlister, C., \& Weakley, A. (2012). Naturalistic assessment of everyday functioning in individuals with mild cognitive impairment: The day-out task. Neuropsychology, 26(5), 631-641.

Singh-Manoux, A., Dugravot, A., Ankri, J., Nabi, H., Berr, C., Goldberg, M., et al. (2014). Subjective cognitive complaints and mortality: Does the type of complaint matter? Journal of Psychiatric Research, 48(1), 73-78.

Slavin, M. J., Sachdev, P. S., Kochan, N. A., Woolf, C., Crawford, J. D., Giskes, K., et al. (2015). Predicting cognitive, functional, and diagnostic change over 4 years using baseline subjective cognitive complaints in the Sydney Memory and Ageing Study. The American Journal of Geriatric Psychiatry, 23(9), 906-914.

Tierney, M. C., Herrmann, N., Geslani, D. M., \& Szalai, J. P. (2003). Contribution of informant and patient ratings to the accuracy of the minimental state examination in predicting probable Alzheimer's disease. Journal of the American Geriatrics Society, 51(6), 813-818. 